\title{
Cubic Spline Extrapolation for Uplink Channel Quality Prediction in LTE-A with Carrier Aggregation
}

\author{
Maria A. Lema, Mario Garcia-Lozano, Silvia Ruiz
}

\begin{abstract}
In the context of wireless communications, an accurate channel state information (CSI) is key for a correct radio resource management. In the uplink of Long Term Evolution Advanced (LTE-A), obtaining up-to-date CSI relies on user equipments (UEs), that send sounding signals throughout the available bandwidth. This procedure is quite challenging when considering the ever increasing bandwidth demands and the introduction of carrier aggregation. Information from many radio resource blocks becomes outdated and unreliable for scheduling operations. Besides, interference values are much more variant than the downlink due to changes in the position of interferers and their transmission power. This work proposes the introduction of cubic spline extrapolation to obtain a prediction horizon that allows extending the reliability of the channel quality evaluation along time. Though very high interference levels and variations thereof imply no gains, the mechanism succeeds with moderate interference, situation that can be provided by interference cancellation strategies. Copyright (C) 2015 John Wiley \& Sons, Ltd.
\end{abstract}

\section{INTRODUCTION}

Mobile traffic has been growing enormously due to the demands for multimedia, social networks and the increasing use of cloud based services. Despite the advances made in the fourth generation (4G) designs, challenging requirements are set to be targeted in the forthcoming fifth generation (5G). Among others, higher system capacity, massive device connectivity and higher data rates are some of the issues to be accomplished by the new 5G technology. To achieve this, the use of carrier aggregation (CA) is mandatory, since provides wider transmission bandwidths, and allows the system to improve the area spectral efficiency and system capacity.

One of the aspects that allows to further increase spectral efficiency is the existence of reliable up-to-date channel state information (CSI), which is also an essential support for many of the transmission procedures. In the uplink (UL) of Long Term Evolution advanced (LTEA) systems, the UL channel response is obtained by the eNodeB (eNB) after receiving and processing the sounding reference signals (SRS) sent by the user equipment (UE), which allows to determine the channel quality information (CQI) [1,2]. Accurate channel state information (CSI) is key to capitalize improvements brought by promising radio resource management (RRM) algorithms: this information can be used at the discretion of the scheduler to support frequency selective scheduling. Sounding signals may be also used to perform effective beamforming in in both UL and downlink (DL), where a correct CSI estimation plays an important role and it is one of the paramount technical issues to be solved in the context of $5 \mathrm{G}$ massive multipleinput-multiple-output (MIMO) systems.

In a system operating with several aggregated component carriers (CCs), the bandwidth to be sounded increases, which complicates the CSI acquisition task. In power limited UEs the power spectral density decreases as the transmission bandwidth increases, which might lead to unsatisfactory CQI measures. CA transmissions in the UL may imply further power limitations to the UE, since maximum power reduction (MPR) is carried out in UL non-contiguous transmissions, to contain the PAPR and hence, the out of band emissions. To avoid inaccurate CQI measurements due to decreased signal power density, LTE allows the eNB to manage sounding radio resources by allocating narrowband SRS transmissions; this allows to effectively obtain reliable sounding measures. In a CA context, this solution can be counterproductive since it rises the time delay between measurements, and channel information becomes outdated and less trustworthy. The eNB should discard frequency parts with outdated SRSs, which yields to a reduction in frequency diversity gains.

A second problem the UL sounding must deal with, is the intrinsic rapid variations of the SINR. This is not only due to short term fading, but also because of scheduling decisions. With every transmission time interval (TTI), allocated resources are updated and the interference perceived on each physical resource block (PRB) changes. 
This implies fast signal to interference noise ratio (SINR) variation and reduces the sounding reliability, eventually generating errors in the link adaptation and reducing the UE throughput.

Given the UL problem and the need for improving CSI to support LTE-A features in CA contexts, we propose a cubic spline extrapolation method to predict the UE SINR within a reasonable prediction horizon. This provides a means for the scheduler to extend the validity of SRS information. The proposal is tested under different interference scenarios, and interference cancellation (IC) has also been considered to support the prediction procedure under harsh interference conditions, and therefore help to further improve the channel estimation. Note that the term channel estimation may also refer to the the estimation of the channel impulse response using the combined knowledge of the transmitted and received signal; assuming the channel affects like a filter, these techniques aim at recovering the received signal by estimating the filter coefficients. In this paper channel estimation refers only to the SINR or CQI estimation at the eNB side, with the aim of improving transmission procedures.

This paper is organized as follows: Next section provides a short survey of the prior work done in this topic, main conclusions of it are described and the research opportunities are detailed. Section 3 provides the reader with some background information regarding the UL sounding system, section 4 describes the main limitations encountered when pursuing a correct CSI acquisition and section 5 describes the proposed solution. The system model is described in section 6 and the evaluation methodology is described in section 7 , followed by the results in section 8 . Finally, this paper ends with the main conclusions of this study, in section 9 .

\section{LITERATURE SURVEY AT A GLANCE}

The 3GPP community has recognised that an adequate management of SRS configuration parameters in LTE-A UL can lead to improvements in the system performance. In [3] authors evaluate the gain of non-contiguous resource allocation in the UL considering two SRS bandwidth setups based on the UE SINR. Results show that a discontinuous allocation may be beneficial as it can exploit additional frequency diversity gains. However, SINR estimation errors provided by SRS inaccuracy or unavailability can lead to degrading effects. Other 3GPP contributions already mentioned the need for different sounding bandwidth options to avoid CQI measurement error. This necessity was already discussed in [4], where authors propose to adaptively control the sounding bandwidth. This solution allows reducing the number of wideband transmissions which assures lower MCS selection error due to decreased received signal power density. Results show that the adaptive control is especially effective in hyper dense scenarios with high volumes of traffic. Also, in [5] different schemes of configuring SRS across multiple CCs are discussed. Authors propose that sounding signals should be configured separately for the different CCs. This turns sounding more flexible, this can be useful in heterogeneous networks interference coordination. Another proposal is that UEs should transmit SRS symbols only in their active CCs, meaning the ones that are actively being used for data transmission.

The channel estimation error in the DL has been tackled by several authors. In [6], authors deal with the channel quality indicator (CQI) delay. This CQI feedback delay is caused by CQI measurement at the UE side, CQI feedback and CQI processing at the eNB side. A polynomial extrapolation method is proposed to predict the CSI, where no second-order statistics of the SINR are required. The solution is evaluated for different UE speeds and results show that the proposed method works for UEs with low variant channels. Another strategy to overcome the performance loss brought by channel estimation error is presented in [7]. Authors propose two types of link adaptation algorithms. The first one performs a weighted average of different past received information of the UE, such as channel, SINR and MCS. The algorithm calculates the current channel, SINR or MCS by averaging the past information in a fixed time window. The performance of the previous algorithm strongly depends on the time window size, therefore, authors propose the use of a dynamically adjusted time window. Link adaptation decisions based on the channel averaging shows to compensate better the channel estimation errors.

Other works that can be found in the literature undertake this problem by using prediction filters. In [8] it is proposed the use Least Mean Square (LMS) prediction method. In particular, a modified version of the normalized LMS is proposed in order to support wider CQI feedback delays. Improvements brought by predictions are very much dependent on the time difference of two consecutive CQI measurements. When the difference is low, the LMS proposed strategy shows significant improvement, however as it increases, the LMS solution degrades the instantaneous channel information. Results conclude that the prediction solution has the ability to improve throughput when the time delay is less than $5 \mathrm{~ms}$. Wienerbased prediction filters are also widely found in the literature as a strategy to overcome the channel estimation errors, [9-11]. This solution has also been proposed for the UL $[12,13]$. Practical Wiener filters are obtained using temporal averages to approximate the cross-correlation and auto-correlation functions involved in the solution and ergodicity therein is also assumed. Work in [14] compares two different approaches in the DL: Wiener filters with cubic spline extrapolation. The cubic spline method constitutes a near optimal solution in low speed UEs and it does not have the strong input/output correlation dependency the Wiener solution has. To adapt the cubic spline solution to fast moving UEs, the authors propose a 
simple heuristic using the autocorrelation of past samples that, combined with the extrapolation output, provides an accurate prediction. Other authors consider Kalman filters for channel prediction in the DL $[15,16]$. In the UL, work in [17] uses Kalman filtering to predict the interferences in a time division multiple access wireless network. These type of networks allow multiple, contiguous time slots to be used by the same terminal or base station for transmitting the data. As a consequence, the interference at the receiver is correlated from one time slot to the next. Based on the interference measurements in previous slots the interference power to be received can be predicted with the use of Kalman filtering. To successfully implement this filter it is crucial to correctly estimate the process noise and measurement noise (both in Kalman terminology, these noises are used in the prediction process), which in general depend on the signal statistics, and in this particular study both noises depend on the error characteristics of the interferences at the receiver.

Specific strategies for the UL may consider collaboration among different eNBs to provide a wider knowledge of the interference map generated, which depends on the resource allocation decision of the eNB. Work in [18] proposes to exchange scheduling decisions between the eNBs, incorporating a time delay that occurs for the information exchange. Each eNB predicts the channels of its own users using a Minimum Mean Square Error (MMSE) predictor and sends the scheduling information in two manners, a fixed allocation decision, or the probability of the scheduling decision. This new scheme provides better results for link adaptation decisions, and in particular, the interchange of probabilities is especially beneficial for fast moving UEs.

\subsection{Remarks from Literature Review}

Table I summarizes the prediction strategies described in the previous section in a comparative way, where the most important features are highlighted. The majority of the work is done in the DL, where the CSI acquisition problems may be similar at a first glance, but the UL indeed presents more challenges, mainly because of the interference variability. There are several proposals that suggest the use of Wiener filtering, which needs second order statistics to compute the filter coefficients.

The Wiener filter, or any linear prediction method that is based on the mean square error (MSE) minimization, require a previous knowledge of the SINR and channel statistics. In general, fading channels can be studied and the second order statistics can be derived, however, doing this while considering interferences is a much more difficult task in the UL, where the variability follows no statistical rule. The same rationale applies for the Kalman filter, where the variances of the process and measurement noise must be estimated as an input. Among the other prediction filters proposed, there is the LMS, which can rule out any low or high order statistics, but however, requires a constant time difference between the past CQI
Table I. Summary of prediction strategies to improve CSI

\begin{tabular}{cccccc}
\hline Ref \# & Link & Strategy & Statistics & Interference & Delays \\
\hline$[6]$ & DL & Polynomial & $\boldsymbol{X}$ & $\checkmark$ & $\checkmark$ \\
{$[7]$} & DL & Average & $\boldsymbol{X}$ & $\checkmark$ & $\checkmark$ \\
{$[8]$} & DL & LMS & $\boldsymbol{X}$ & $\boldsymbol{X}$ & $\checkmark$ \\
{$[9]$} & DL & Wiener & S.O & $\boldsymbol{X}$ & $\boldsymbol{X}$ \\
{$[10]$} & DL & Wiener & S.O & $\boldsymbol{X}$ & $\boldsymbol{X}$ \\
{$[11]$} & DL & Wiener & S.O & $\boldsymbol{X}$ & $\boldsymbol{X}$ \\
{$[12]$} & UL & Wiener & S.O & $\boldsymbol{X}$ & $\checkmark$ \\
{$[13]$} & UL & Wiener & S.O & $\boldsymbol{X}$ & $\boldsymbol{X}$ \\
{$[14]$} & DL & Polynomial & X & $\checkmark$ & $\checkmark$ \\
{$[15]$} & DL & Kalman & L.O & $\checkmark$ & $\checkmark$ \\
{$[16]$} & DL & Kalman & S.O & $\checkmark$ & $\checkmark$ \\
{$[17]$} & UL & Kalman & L.O & $\checkmark$ & $\checkmark$ \\
{$[18]$} & UL & MMSE & S.O & $\checkmark$ & $\checkmark$ \\
\hline
\end{tabular}

S.O: Second Order $\quad$ L.O: Low Order

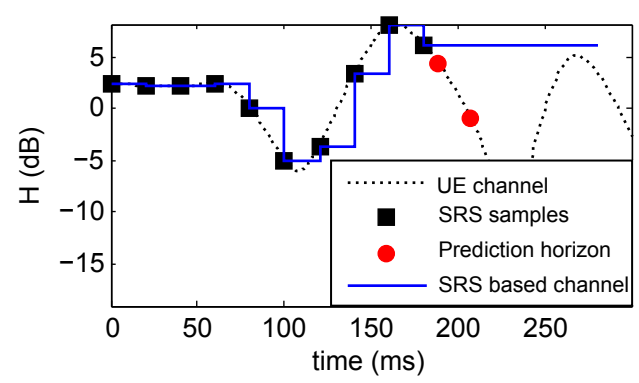

Figure 1. Continuous time formulation of the prediction

samples and the prediction. In the UL, the SRSs in a particular piece of spectrum are only available after a complete bandwidth sweep, but the scheduler may need to decide about the suitability of those resources at any time. So, a continuous time formulation for the prediction is required, which is mathematically tricky for Wiener based solutions. The main challenge of the selected prediction method is to provide the most accurate SINR at the time of reception, and this decision shall be made in the scheduling instant. In particular, the time span for predictions goes from one round trip time (RTT), concurring with a SRS reception, to one RTT plus the delay in SRS reception, this instant is the immediate one prior to the SRS reception, figure 1 shows a graphical example of this. In a CA context the delay in the SRS reception can be seriously affected, since larger bandwidths need to be sounded.

The proposed method, is a numeric function that is piece-wise defined by several polynomial functions, in the particular case of cubic splines, degree three polynomials. The spline can be used for prediction by extrapolating the last polynomial function, and obtaining a future value of the curve, which is, in this case, built upon the past SINR samples. In this sense, the use of splines 
constitutes a much different approach compared to the previous strategies, this mathematical method does not filter the signal, and it can approximate functions with less computational complexity, as shown in [19]. The cubic spline extrapolation method avoids complex error calculations, as in the Wiener case where the obtention of second order statistics are mandatory; also, since prediction is not in a discrete form as in the previous cases, the curve provides broader information about its behaviour in the future.

\subsection{Research Efforts and Main Contributions}

From the previous paragraphs, a mathematical method based on cubic splines appears to be an interesting and promising solution that suits well the constraints of the problem, in particular:

1. The difficulty to estimate error measurements correctly in the uplink, given the rapid SINR variability.

2. It is a continuous time formulation itself, without the need for additional interpolators.

Frequency domain scheduling and link adaptation decisions are supported by the SINR information provided by the SRS. Because of the interference variability, the time difference between the SRS and data reception, and UE transmit power considerations, there is a misalignment between the SINR measured from the SRS and the actual one measured at the physical uplink shared channel (PUSCH) transmission. In this sense, CSI acquisition techniques must place efforts in reducing the SINR misalignment and thereby improving the system performance.

Therefore, the main contribution of this work is to increase the SRS reliability considering:

- Realistic interference generation: The generation of UL interferences variate with time mainly due to scheduling decisions.

- SRS delays: Not only affected by the RTT, but also because of hopping along the entire band. Delays are much aggravated with the use of aggregated carriers.

- Low complex prediction methods.

- IC techniques. Used in the eNB side to suppress the most dominant interference sources, and hence, reduce both the average interference and its variability.

\section{CHANNEL QUALITY INDICATOR ACQUISITION IN THE UPLINK}

Sounding signals are sent by the UE and configured by the eNB with the main goal of achieving up-to-date and accurate CQI. This allows performing opportunistic frequency domain scheduling, since the best spectrum

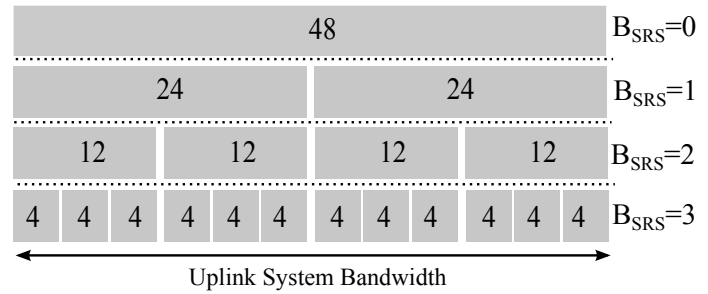

Figure 2. Sounding reference signal bandwidth configuration example for one $C_{\mathrm{SRS}}$, expressed in PRBs

areas can be detected. Eventually it also contributes in the decision of the best modulation and coding scheme (MCS) in the link adaptation process. SRSs are sent in the last single-carrier orthogonal frequency division multiple access (SC-FDMA) symbol of the sub-frame, only one out of seven symbols are reserved for sounding purposes, and the reference signal bandwidth is configurable. Sounding signals can be as frequent as every second sub-frame or as infrequent as every 32th sub-frame. The eNB is in charge of selecting both cell and UE specific sounding parameters with the objective of achieving up-to-date CQI without increasing interferences, and accounting for the UE power headroom. All the sounding parameters such as maximum sounding bandwidth or frequency position are configured on a cell-wide basis. Specific perUE configuration parameters are: sounding periodicity, specific sounding bandwidths, and hopping patterns.

The maximum sounding bandwidth, $C_{\mathrm{SRS}}$, is a cell-specific parameter signalled via radio resource control (RRC) messages. A range of $C_{\mathrm{SRS}}$ bandwidth configurations are available depending on the system bandwidth, these are defined in [20] in number of PRBs. For each $C_{\text {SRS }}$ selected by the eNB, four different UE specific bandwidths are possible, $B_{\mathrm{SRS}}$, on the basis of a tree structure. This allows the sounding region to be configured to span only in the resource blocks used, as for example, in those ICIC schemes in which UEs are restricted to transmit in certain parts of the spectrum. Also, narrow bandwidth soundings are desirable for power limited UEs, since an increase in bandwidth reduces its power spectral density (PSD), which leads to less accurate SINR measurements [2]. Figure 2 shows a graphical example of the different $B_{\mathrm{SRS}}$ values that can be configured for one $C_{\mathrm{SRS}}$, expressed in number of PRBs. If the $C_{\mathrm{SRS}}$ selected by the eNB is $48 \mathrm{PRBs}$, then the UE may sound the entire band in sets of 4,12, 24 or 48 PRBs.

As said, the $B_{\mathrm{SRS}}$ of UEs placed at the cell edge should be selected considering their low power availability to assure the reliability of the measurement. But, if the sounding bandwidth is narrow, the time to measure the entire system bandwidth is larger, increasing the period between two consecutive measurements of the same piece of spectrum $\left(T_{\text {sound }}\right)$, as shown in figure 3 . This is particularly problematic in carrier aggregated systems making use of very wide bands. 


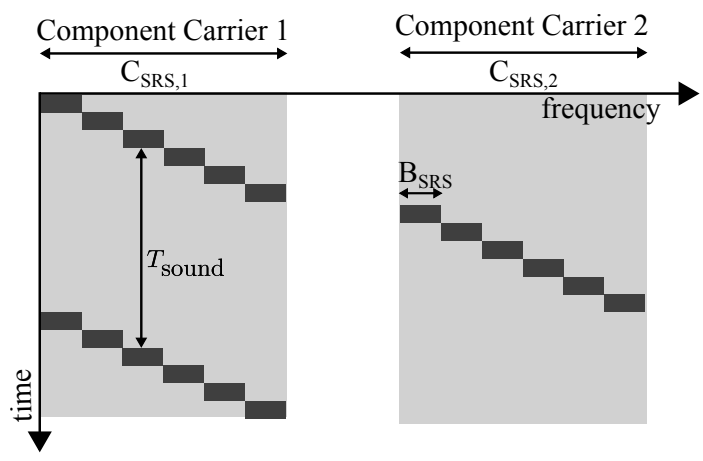

Figure 3. Sounding procedure all UEs in the scenario follow

Both Frequency and Code Division Multiplex (FDM and CDM) are used in LTE to support a higher number of users when sounding. FDM is done following a transmission comb structure with a factor of 2 , which means that only every other subcarrier is sounded, and $\mathrm{CDM}$ is done using a Zadoff-Chu sequence with different cyclic shifts. For each sounding region up to eight UEs can be multiplexed via cyclic shifts, $n_{\mathrm{SRS}}$. Based on this, the combination of FDM and CDM allows to multiplex a total of $16 \mathrm{UEs}$ in the same spectrum area. In practice, this number of UEs is not feasible since there are orthogonality and interference issues, so a more realistic estimate is to multiplex 6 to 8 UEs [2].

Following this sounding procedure, the eNB is able to measure the uplink channel response. Interferences from other reference signals can be contained with the use of orthogonal code sequences; the cross-correlation of simultaneous eNB transmission is reduced, thus reducing inter-cell interference.

\section{PROBLEM STATEMENT}

The number of users sharing the spectrum as well as the sounding bandwidth $B_{\text {SRS }}$ impact the delay in the channel measurement, also the use of aggregated carriers has an impact on it. Table II shows the time delay $T_{\text {sound }}$, in milliseconds, experienced between two consecutive sounding measurements in one frequency resource block. This time delay varies depending on the number of users connected to the eNB and the piece of bandwidth being sounded each TTI, $B_{\text {SRS }}$. Delays shown also account for the use of two CCs (20 MHz each), and are compared to a single carrier (SC) scheme. Each carrier is sounded separately on different transmission intervals, to avoid further power reductions inflicted by non-contiguous transmission in the UL [21]. The delay figures show that with CA it increases proportionally with the number of carriers aggregated. The main problem of having increased delays in the channel measurement is the lack of up to date information in the scheduling decisions and link adaptation. Figure 4 shows two different examples of

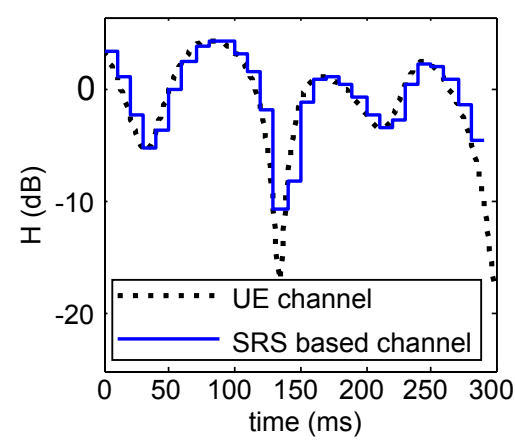

(a) UE Channel measured by SRS with time delay of $10 \mathrm{~ms}$

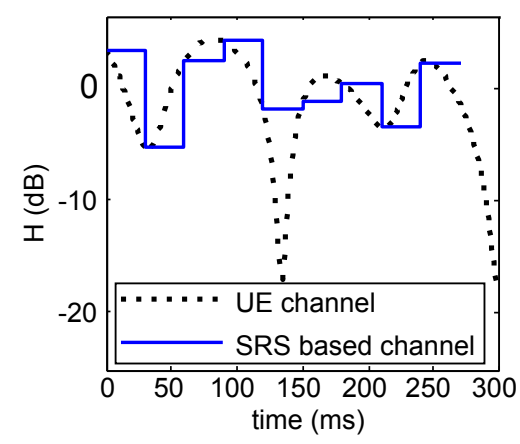

(b) UE Channel measured by SRS with time delay of $30 \mathrm{~ms}$

Figure 4. Channel measurement for two different $T_{\text {sound }}$

channel state measurement with the use of SRS. The first figure, 4(a), has a time delay between two samples of $10 \mathrm{~ms}$ and the second one, figure 4(b), has a time delay of $30 \mathrm{~ms}$. A lower time delay can capture enhanced channel information in terms of instantaneous deep fadings.

Another limiting aspect of the UL channel measurement is its intrinsic interference variability, which impacts the CQI by constantly altering the aggregate interference level experienced. According to the scheduling decisions, interferer UEs may change from one transmission time to another, and the impact on the SINR depends on the larger or lesser the pool of candidates is: the former generates increased interference variability compared to the latter. Figure 5 shows the SINR measured over the PUSCH for two different schedulers: round robin (RR) and proportional fair (PF). The simulation here is quite simple for the RR case, UEs are always allocated in the same order and sequentially, given that the number of available resource blocks is higher than the number of UEs allocated, UEs are always allocated the same resources, resulting in a les variable interference (since interferers are always the same). In the PF case the scheduler separates the process in two parts, a time domain part, where UEs are ordered based on their past acknowledged throughput, and a frequency domain part, where PRBs are allocated with the goal of maximizing the cell spectral efficiency. 
Table II. Time delay in milliseconds between two consecutive SRS measurements $\left(T_{\text {sound }}\right)$. Two CC of $20 \mathrm{MHz}$ each.

\begin{tabular}{|c|c|c|c|c|c|c|c|c|c|c|c|c|}
\hline \multirow{3}{*}{ Number of UEs } & \multicolumn{12}{|c|}{$B_{\text {SRS }}$} \\
\hline & \multicolumn{2}{|c|}{4} & \multicolumn{2}{|c|}{8} & \multicolumn{2}{|c|}{16} & \multicolumn{2}{|c|}{20} & \multicolumn{2}{|c|}{40} & \multicolumn{2}{|c|}{80} \\
\hline & $\mathrm{CA}$ & $\mathrm{SC}$ & $\mathrm{CA}$ & $\mathrm{SC}$ & $\mathrm{CA}$ & $\mathrm{SC}$ & $\mathrm{CA}$ & $\mathrm{SC}$ & $\mathrm{CA}$ & $\mathrm{SC}$ & $\mathrm{CA}$ & SC \\
\hline 8 & 40 & 20 & 20 & 10 & 10 & 5 & 8 & 4 & 4 & 2 & 2 & 1 \\
\hline 24 & 40 & 20 & 20 & 10 & 10 & 5 & 8 & 4 & 4 & 2 & 4 & 2 \\
\hline 40 & 40 & 20 & 20 & 10 & 10 & 5 & 8 & 4 & 8 & 4 & 6 & 3 \\
\hline 72 & 40 & 20 & 20 & 10 & 10 & 5 & 16 & 8 & 12 & 6 & 8 & 4 \\
\hline
\end{tabular}

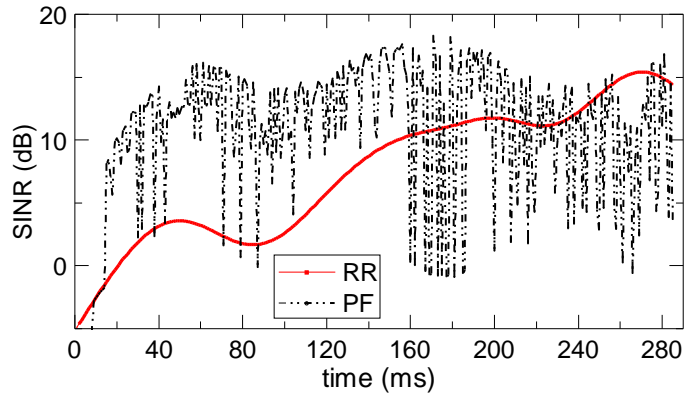

Figure 5. SINR evolution for RR and PF schedulers.

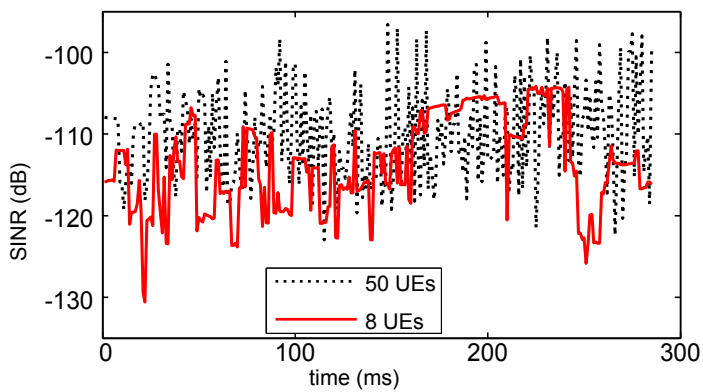

Figure 6. SINR evolution for two cell occupancies

In this regard, the PF scheduler provides more changeable allocations which influences the interference variability.

The variability in time of the interference generated is highly impacted by the number of UEs having active connections at the eNB: the higher the number of UEs, the more variable the interference becomes. The probability of having always the same interferer source is lower as the number of connections increase. Figure 6 shows the evolution of the SINR over time for two different eNB occupancies. When the cell occupation is low, the interference variation over time results in a less changeable SINR, while increasing the load in the cell leads to increased variations of the SINR.

Due to the temporal variability of the SINR and the increased delays in sounding experienced in high bandwidth transmissions, accurate CSI acquisition becomes challenging. The main goal is to find a method to support the use of SRSs in high reporting interval situations, motivated by the use of CA, that is able to improve the $\mathrm{CQI}$ reliability under realistic interference situations.

\section{PROPOSED SOLUTION}

The fundamental idea behind the cubic spline is getting a set of smooth curves through a number of predefined data points [22], in this case the SINR measured from previously received SRS. So given a past observation time in the interval $[a, b]=\left\{a=x_{0}<x_{1}<\cdots<x_{m}=b\right\}$, the SINR prediction function $\mathcal{S}(x)$ is a third order spline and satisfies the form:

$$
\mathcal{S}(x)= \begin{cases}s_{1}(x) & \text { if } x_{0} \leq x<x_{1}, \\ s_{2}(x) & \text { if } x_{1} \leq x<x_{2}, \\ \vdots & \\ s_{m}(x) & \text { if } x_{m-1} \leq x<x_{m}\end{cases}
$$

where each $s_{i}(x)$ is a third order polynomial defined by:

$$
s_{i}(x)=a_{i}\left(x-x_{i}\right)^{3}+b_{i}\left(x-x_{i}\right)^{2}+c_{i}\left(x-x_{i}\right)+d_{i} .
$$

To calculate the unknown coefficients of the spline function $4 \cdot m$ equations are required. They can be posed considering the following properties:

1. $\mathcal{S}(x)$ must be contiguous on its entire interval, $s_{i}\left(x_{i}\right)=s_{i+1}\left(x_{i}\right)$. This means that each polynomial goes through two consecutive data points, obtaining $2 \cdot m$ equations.

2. To make $\mathcal{S}(x)$ smooth across the intervals its first and second derivative $S^{\prime}(x) S^{\prime \prime}(x)$ are also contiguous. This results in $2 \cdot(m-1)$ equations.

3. Finally, the boundary conditions provide the two missing equations. In this case a natural spline is assumed, $\mathcal{S}^{\prime \prime}\left(x_{0}\right)=0, \mathcal{S}^{\prime \prime}\left(x_{m}\right)=0$.

Figure 7 depicts the explained mathematical method. Figure 8 shows how the spline correctly matches an extended pedestrian B (EPB) channel model beyond one round trip time (RTT) of $8 \mathrm{~ms}$; the figure shows the channel impulse response, where $H$ is $\log \left(|H|^{2}\right)$. This qualitative 


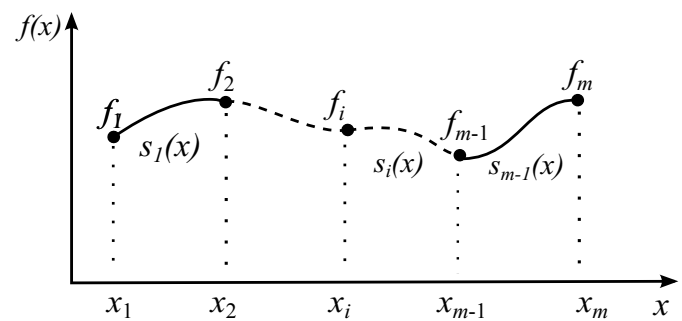

Figure 7. Cubic spline interpolation of data points.

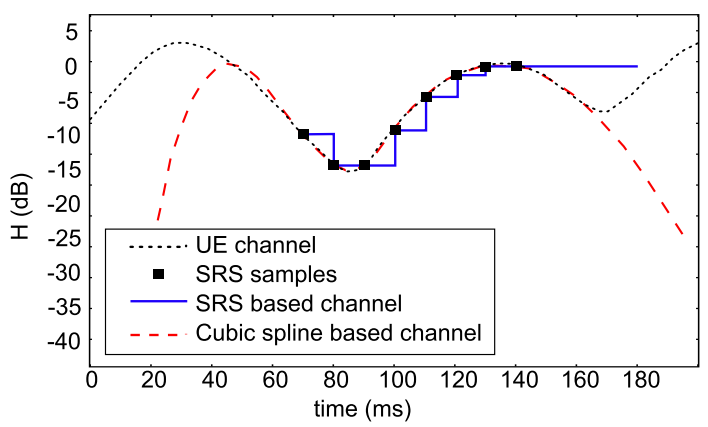

Figure 8. Channel prediction cubic spline extrapolation

analysis shows a superior channel evaluation than just keeping constant the last SRS information (SRS based channel on the plot). Note however, that interference is not present yet. The squares indicate the reporting points or times in which the eNB receives an SRS from the UE, $10 \mathrm{~ms}$ in this example. It is remarkable that no matter how often SRS are received, if the user is allocated that resource, it will be being used one RTT later in the best case, so at least that prediction horizon should be aimed. Since the PRB is the minimum allocable unit in LTE, the eNB just needs to compute a CQI per PRB or even small sets of them, so an average among all the channels per subcarrier is performed. The presence of interference yields a much noisier evolution of SRSs, so the resulting spline is expected to have sharper variations. Figure 9 shows an example of prediction in case of a channel affected by interference from users in neighbouring cells. It is evident how the presence of large interference variations from one TTI to the next may imply that the spline is not able to follow the channel and so wrong scheduling decisions can be taken about the quality and suitability of particular PRBs. Moreover, in some cases the first derivative at the last SRS reporting point can be very high and so the duration of the reliable prediction horizon is dramatically reduced. Hence, it is necessary to introduce a divergence detection mechanism. The spline is considered to diverge when variations higher than $10 \mathrm{~dB}$ occur in less than one RTT, this simplistic heuristic is enough to filter out those cases. Then the last prediction that was considered as reliable is kept fix until a new SRS update.

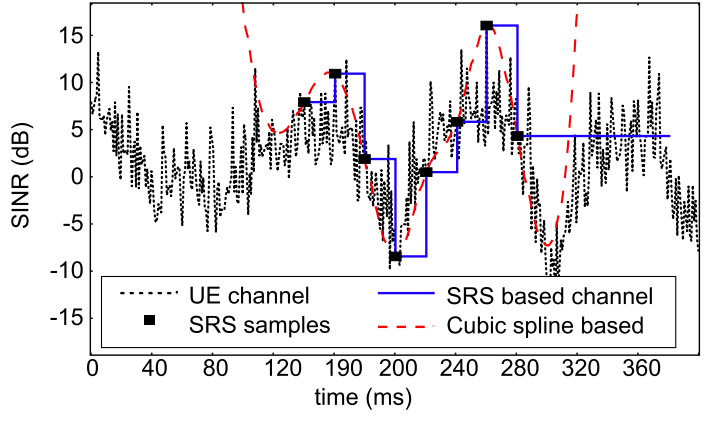

Figure 9. UE channel prediction based on cubic spline extrapolation, effect of low SINR.

\section{SYSTEM MODEL}

We consider a LTE-A UL system with CA, consisting of $C$ aggregated CCs. The criteria for selecting the UEs that perform CA is based on their available power [23].

Consider a macro cell network with a set of $\mathcal{J}$ eNBs, each one has a total number $\mathcal{I}$ of active users. All CCs are in the same frequency band with the exact same bandwidth, and each one is comprised of $\mathcal{R}$ PRBs which are allocated to users each TTI. Each user, is assigned a user block $u b$ and a cyclic shift $n_{m}$ (where $m \in M$ and $M$ is the number of multiplexed UEs). All UEs with the same $u b$ share the same allocation, therefore different cyclic shifts $n_{m}$ are assigned. The eNB allocates groups of successive resource blocks to each user block; in one allocation process it distributes the total bandwidth along all the user blocks.

Open loop power control (OLPC) expressed in equation 3 determines the UE transmitted power for a CA system considering non-contiguous resource allocation within one $\mathrm{CC} ; \pi_{A}$ corresponds to the maximum power reduction (MPR) that is intrinsic to non-contiguous allocation, introduced to contain non-linearities of the power amplifier [24]; $P_{0}$ and $\alpha$ are OLPC parameters [25] and $L$ is the UL path-loss.

$$
P_{\mathrm{TX}}^{\mathrm{MC}}=\min \left(P_{\max }-\pi_{A}, P_{0}+10 \log M_{\mathrm{Alloc}}+\alpha \cdot L\right)
$$

The eNB allocates a set of $M_{\text {Alloc }}=M_{\text {SRS }}$ contiguous PRBs to be sounded and obtain a CQI measurement. Note that to avoid further power reductions, no simultaneous SRS transmission in both CCs is considered, therefore when calculating the SRS transmission power, $P_{\mathrm{SRS}}, \pi_{A}$ is set to zero. The signal received per PRB $r$ at the eNB side in its $c^{\text {th }} \mathrm{CC}$ and one RTT later is denoted as $S_{c, r}^{\mathrm{UL}}(i, j)$. For the sake of simplicity subindex $c$ has been omitted in all equations.

$$
S_{r}^{\mathrm{UL}}(i, j)=P_{\mathrm{SRS}} h_{r}(i, j) d(i, j)^{-\alpha} 10^{\frac{\chi}{10}}
$$

Where $P_{\mathrm{SRS}}$ is the SRS transmit power, calculated with equation $3 ; h_{r}(i, j)$ is the Rayleigh fading at PRB level; $d(i, j)$ is the distance from user $i$ to the eNB $j$ and $\alpha$ is the path loss exponent; $\chi$ is a Normal random variable with zero mean and standard deviation $\sigma$. 
With this, the eNB can estimate the user's $i$ SINR at $\mathrm{PRB} r$ in carrier $l$ as:

$$
\gamma_{r}^{\mathrm{SRS}}(i, j)=\frac{S_{r}^{\mathrm{UL}}(i, j)}{I_{r}(i, j)+\sigma_{n}^{2}}
$$

where $\sigma_{n}^{2}$ is the noise power and $I_{r}$ is the total aggregate inter-cell interference perceived in PRB $r$ which is modeled as:

$I_{r}(i, j)=\sum_{n \in N} P_{\mathrm{SRS}, n} h_{r}(i, j) d(i, j) d(n, j)^{-\alpha} 10^{\frac{\chi}{10}}$

where $N$ is the set of interfering UL users associated to the neighbouring cells.

The SINR in equation 5 is the one providing the CQI used by the eNB for transmission procedures. This value of SINR is updated when a new SRS reporting is available, i.e. every $T_{\text {sound }}$.

Following this sounding process along the entire CC (all PRBs in $\mathcal{R}$ ) lets to obtain a single value of $\gamma_{r}^{\mathrm{SRS}}(i, j)$ for every PRB. The scheduler's main task is to allocate $M_{\text {Alloc }}=M_{\text {PUSCH }}$ in order to maximize throughput while maintaining a proportional fairness in the coverage area. Based on the average $\overline{\gamma^{\text {SRS }}(i, j)}$ over the selected PRBs, the eNB signals the UL scheduling grant.

The received PUSCH signal one RTT later is:

$$
S^{\mathrm{UL}}(i, j)=P_{\mathrm{TX}}^{\mathrm{MC}} h(i, j) d(i, j)^{-\alpha} 10^{\frac{\chi}{10}}
$$

where $P_{\mathrm{TX}}^{\mathrm{MC}}$ is obtained from the OLPC formula with the corresponding maximum power reduction for CA allocations, following equation 3 . The resulting SINR is:

$$
\gamma^{\mathrm{PUSCH}}(i, j)=\frac{S^{\mathrm{UL}}(i, j)}{\overline{I(i, j)}+\sigma^{2}}
$$

where $\overline{I(i, j)}$ is the average interference perceived in all $M_{\mathrm{PUSCH}}$. The interference in the PUSCH channel is modelled as in the SRS channel.

From equations 5 and 8 it is inferred that a difference in both may arise: difference in interference perception, because sources of interference change with time, and also differences in the received power due to the channel impulse response evolution with time. The prediction method calculates the SINR $\gamma^{\mathrm{PUSCH}}(i, j)$ at the time of reception $t_{p}=t+1 R T T$, where $t$ is the scheduling instant. In particular, the prediction horizon time span, with respect to the last SRS reporting instant, goes from $t_{p}=1 R T T$, concurring with the SRS reception, to $t_{p}=T_{\text {sound }}-1+1 \mathrm{RTT}$, when the prediction horizon corresponds to one TTI prior the arrival of the next SRS.

Finally, a simple successive IC process is considered to clean the desired signal from the strong temporal variations. The basic concept of this strategy is that the interference power can be reduced by decoding and subsequently subtracting the interference signals. IC is

\begin{tabular}{|c|c|}
\hline Parameter & Value \\
\hline Inter-site distance & $500 \mathrm{~m}$ \\
\hline Bandwidth & $2 \times 20 \mathrm{MHz}$ \\
\hline Power delay profile & Extended Pedestrian-B \\
\hline Doppler model & Young and Beaulieu $3 \mathrm{~km} / \mathrm{h}$ \\
\hline Shadowing correlation distance & $50 \mathrm{~m}$ \\
\hline Shadowing deviation & $6 \mathrm{~dB}$ \\
\hline$B_{\mathrm{SRS}}$ & 16 PRBs \\
\hline$T_{\text {sound }}$ & $10 \mathrm{~ms}$ \\
\hline Number of UEs served & 10 and 15 \\
\hline Number of UEs connected & 10 and 30 \\
\hline PRBs allocated & 2 clusters of 2 PRBs $x$ CC \\
\hline Simulation time & $10 \mathrm{kTTI}$ \\
\hline UE Buffer size & $1 \mathrm{Mb}$ \\
\hline Round trip time & $8 \mathrm{~ms}$ \\
\hline Target BLER & $10 \%$ \\
\hline Maximum UE transmission power & $23 \mathrm{dBm}$ \\
\hline
\end{tabular}
applied in both SRS and PUSCH received signals.
Table III. Simulation scenario assumptions

\section{EVALUATION METHODOLOGY}

The mechanism is evaluated in a highly realistic dynamic system level simulator which follows the guidelines in [1, 21]. The simulation scenario is a macro-cell case with 14 sites and 3 sectors per site. An EPB channel model is used, considering a UE speed of $3 \mathrm{~km} / \mathrm{h}$. Spatially correlated log-normal variations are introduced. 3D radiation patterns from commercial antennas have been used with $10^{\circ}$ electric tilt adjustment. Two contiguous CCs of $20 \mathrm{MHz}$ each are configured, resulting in a total bandwidth of $40 \mathrm{MHz}$. Link layer abstraction is performed with the classic mutual information effective SINR mapping.

Users are uniformly random spread with finite buffer sizes. Once the buffer is empty, the UE is automatically reconnected in a different part of the same cell, so that the number of connections remain constant. Uplink open loop power control parameters are adjusted following [25] and are equal for all eNB and CCs. Further details about simulation assumptions are listed in Table III. With the aim of testing the proposed strategy under different interference situations three scenarios are studied:

- A low resource utilization (RU) scenario where 10 UEs have an active connection to each cell and $50 \%$ of the resources are actively used.

- Higher number of UEs, 30 have an active connection with $50 \%$ of the resources being actively used.

- High number of active connections 30 UEs and $75 \%$ RU.

The allocated resources per UE remain constant on each studied scenario and the RU varies depending on the number of UEs that are allowed to access the scheduler. 


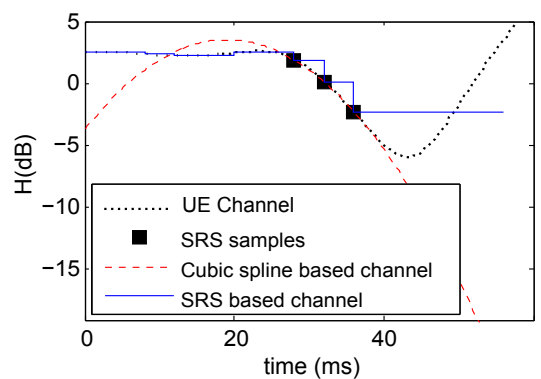

(a) Cubic spline with 3 SRS samples

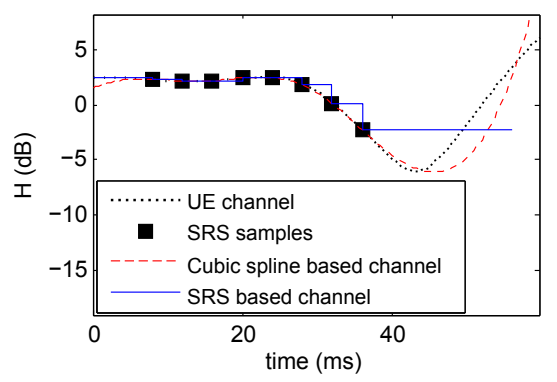

(c) Cubic spline with 8 SRS samples

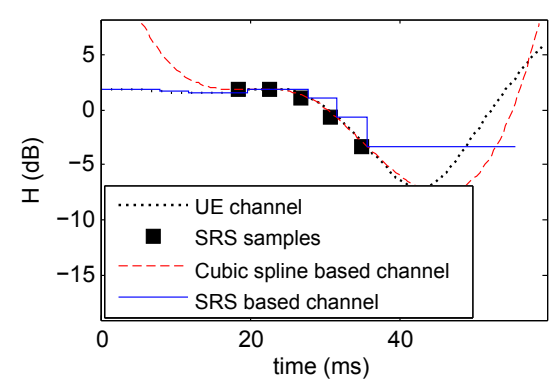

(b) Cubic spline with 5 SRS samples

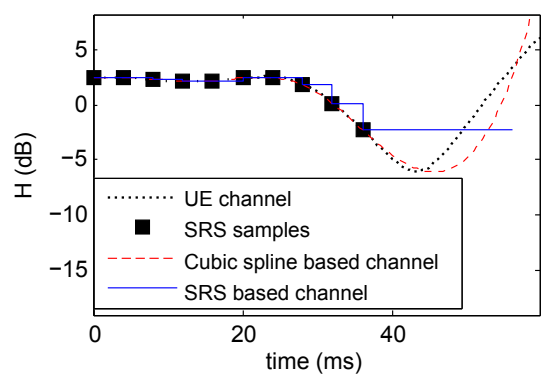

(d) Cubic spline with 10 SRS samples

Figure 10. Cubic spline construction with different number of SRS samples

There is a fixed number of UEs allocated by the frequency domain scheduler at each TTI [26].

The selected method, cubic splines, is compared to a linear extrapolation method, in which the last two SRS samples are used to calculate the future sample, and to the baseline scenario, where the scheduler just uses the SINR at the last SRS for the allocation and link adaptation.

\section{RESULTS AND DISCUSSION}

\subsection{Impact of Spline size}

As explained in section 5, the piece-wise polynomial method needs SINR points measured from the SRS to interpolate and construct the whole curve. The number of past SRSs used for this purpose has an impact in the extrapolation result, figure 10 shows the curve construction with different number of sampling points.

The minimum number of points to construct a curve is three, and as the number increases, more polynomials are considered in the cubic spline construction which leads to different results. The cubic spline depicted in the figure shows that the piece-wise curve achieves a maximum point, in which by adding more polynomials no difference is recognised. In this study the spline construction achieves a state of no further change when more than 8 SRS samples are considered, and this value is considered to conduct the performance evaluation.

\subsection{Error Distribution}

The performance of the CSI acquisition methods are evaluated with the pdf of the SINR error, or misalignment. This error is measured as the difference between the SINR measured in the PUSCH transmission and the sounded or predicted signal used for scheduling and link adaptation. Let recall that the prediction instant, or prediction horizon, can go from one RTT, in the case when the SRS has just been received in the current TTI, or $T_{\text {sound }}-1+1$ RTT in the worst case.

Figure 11 shows the error of the CQI acquisition process in the presence of interference. In particular, it shows the pdf of the SINR error for the three interference scenarios simulated. Interference variations lead to strong errors in the channel measurement; in all the scenarios evaluated the probability of error zero never surpasses $35 \%$ for the baseline case, and predictions perform clearly worse. Under such conditions it is not possible to improve the CSI, because not even the SRSs are providing sufficient information to correctly reconstruct the SINR curve. The interference variability is the main limitation of the channel estimation based on prediction or sounding. In conditions where the UE is exposed to high interference variations it is very difficult to predict the interference behaviour in the forthcoming TTIs, the variability that the interference 


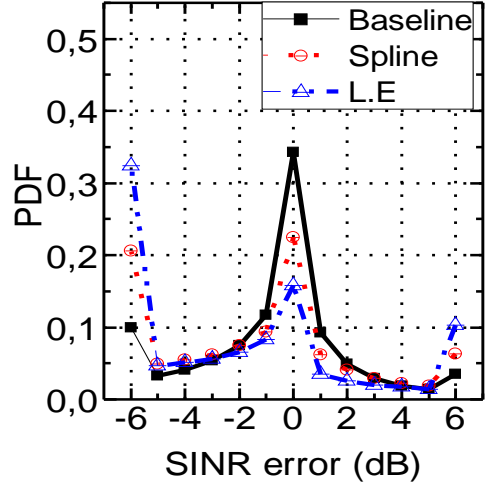

(a) $10 \mathrm{UEs}$ and $\mathrm{RU}=50 \%$

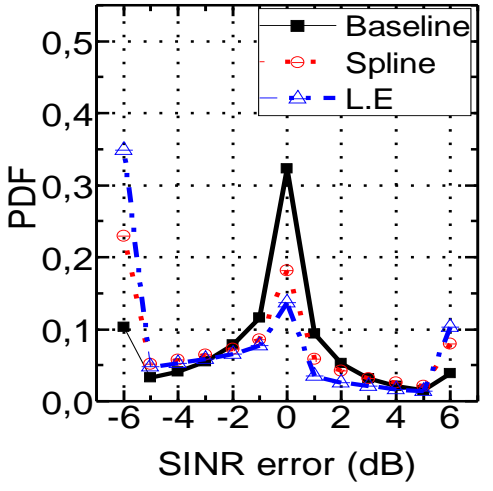

(b) 30 UEs and RU=50\%

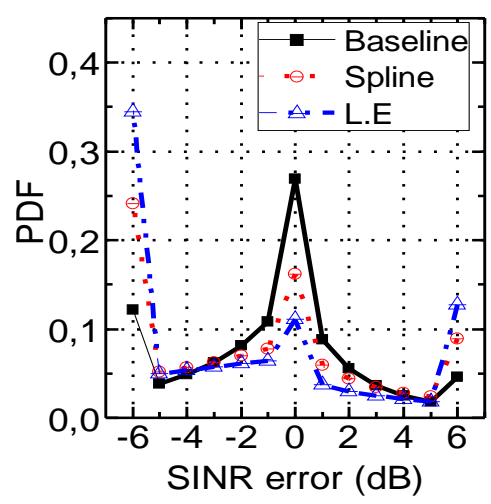

(c) 30 UEs and RU=75\%

Figure 11. SINR error distribution for the different evaluated scenarios in normal interference conditions

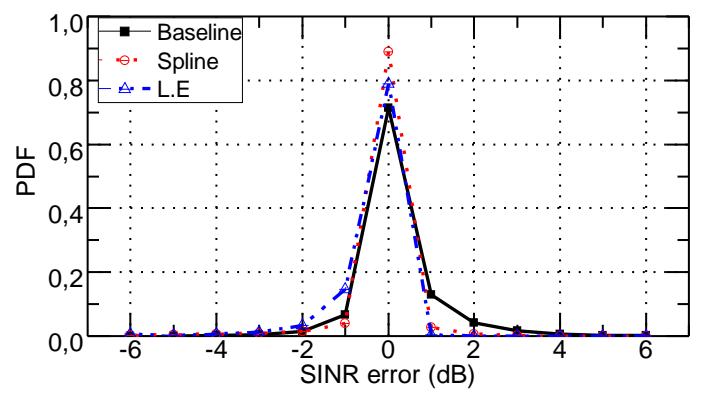

Figure 12. pdf of SNR error measurement.

causes to the SINR can have a dynamic range higher than $10 \mathrm{dBs}$. In this sense, it is more profitable to maintain the past value than to predict the SINR behaviour. However, the probability of error zero in this case is very low, despite the cell occupancy and resource utilization.

\subsection{Impact of Interference Cancellation}

With the aim of improving the SRS information, and also to support predictions and obtain higher probability of null error, IC strategies are introduced. An initial study of signal to noise ratio (SNR) prediction is represented in figure 12, where it shows that the prediction method can comfortably improve CSI. In this case, the number of connections and the RU are irrelevant given that only the SNR is measured (i.e. no interference is considered). There is almost $20 \%$ of improvement in the null error probability with the use of splines, and also errors between $-2 \mathrm{~dB}$ and $2 \mathrm{~dB}$ are reduced. This shows that there is a wide room for improvement with the use of cubic splines, that outperform also the linear extrapolation case. However, the complete removal of interferences is complex and no IC receiver is capable of doing this, such an assumption would not be realistic. Therefore, an intermediate scenario shall be encountered where the IC can provide significant improvements to the SINR prediction and the CSI acquisition itself. Figure 13 shows the percentage of null SINR error under different IC conditions. The interference cancelled is expressed in terms of interference sources; information about the amount of $\mathrm{dBs}$ cancelled is not provided since the most dominant interference source affecting to the different UEs may have tenths of $\mathrm{dBs}$ of difference, hence, no absolute value of $\mathrm{dBs}$ level can be provided.

From the figure, it is noted that each interference scenario has a different minimum cancellation threshold. For instance, in the low load case if two sources are cancelled the cubic spline offers a $2 \%$ improvement over the baseline case, and this improvement boosts as the number of cancelled interferences grows. The linear extrapolation method provides poorer performance compared to the polynomial solution, however it also offers some improvements as the interference variability is reduced. When the number of connections increase and the RU is kept at $50 \%$, three cancelled sources are necessary to provide improvement with the spline method, and when the RU increases to $75 \%$ four sources are needed to be cancelled. This performance is expected, since the number of UEs connected and the RU affect directly to the interference generation and variability.

It is interesting to note, that in all the interference scenarios studied, for a number of cancellations higher than two the baseline scenario does not offer much improvement and arrives to a saturation point, while in the prediction case the improvement continues to grow. Apart from the interference impact, it is recognised that the baseline case presents an upper bound limit in its performance, which cannot be further improved by removing sources of interference. In fact, the saturation point is not far from the performance obtained in the absence of interference; this upper bound limit the SRS has is very much related to the sounding delay. Note that the divergence control mechanism added to the spline 


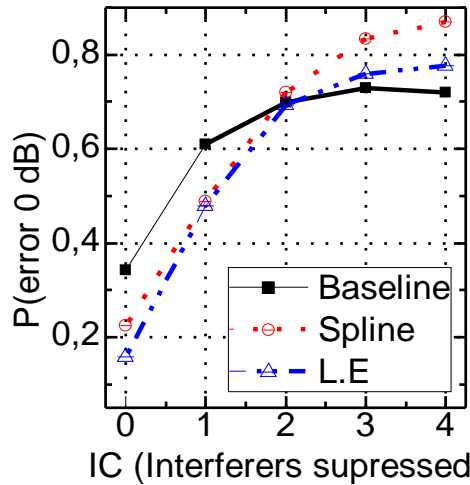

(a) $10 \mathrm{UEs}$ and $\mathrm{RU}=50 \%$

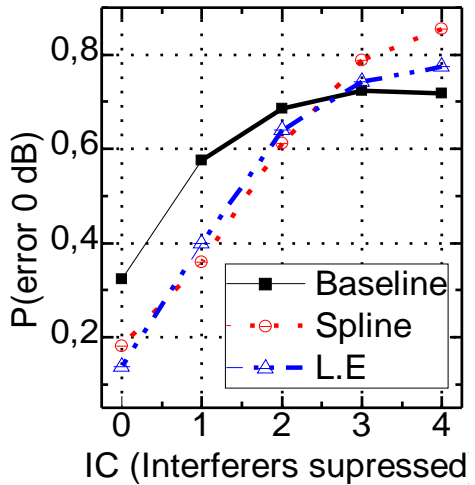

(b) 30 UEs and RU=50\%

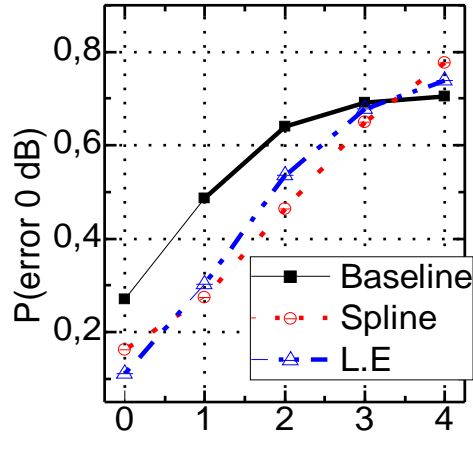

IC (Interferers supressed

(c) 30 UEs and RU $=75 \%$

Figure 13. Probability of null SINR error versus the number of sources of interference cancelled

prediction method helps not to increase huge errors in the SINR prediction, and not to grow it more than the one already provided by the SRS.

\section{CONCLUSIONS}

This paper has addressed one of the main problems that the UL of LTE-A may encounter when pursuing good spectral efficiency and low BLER transmissions. Imperfect CSI acquisition and knowledge impairs the correct performance of other auspicious RRM algorithms, because it entails increased errors and retransmissions. The UL channel presents two main challenges to improve the CQI accuracy, first, the ever-changing interference that results from variable scheduling decisions, and second, the delay in channel reporting. In LTE-A the UE sends SRSs every sub-frame to provide a complete knowledge of the SINR across the entire available band, and this information is useful to support frequency domain scheduling decisions and accurate link adaptation. Thus, increased time-variations of the SINR values and large delay between two consecutive measurement reports can be detrimental for the correct UE performance.

With the aim of improving the SINR knowledge, this paper has proposed the use of a cubic spline extrapolation method that allows extending the SINR knowledge between the reception of two SRSs. The proposed strategy can be applicable under realistic CSI acquisition conditions. It has been evaluated under different interference situations and compared against the baseline and other benchmark solutions. The cubic spline extrapolation method has shown room for improvement of the SINR misalignment when interference variability is kept low with the use of IC. It has been seen that the CSI acquisition can be severely damaged in high interference variability conditions, therefore, this would be a feasible solution only if a certain level of IC is performed. The level of IC has been accordingly quantified, and the proposed solution presents almost a linear improvement with respect to the level of interference cancelled, while in the baseline scenario it has been recognised an upper limit inflicted by the SRS delay.

\section{ACKNOWLEDGMENT}

This work was supported by the Spanish National Science Council through the project TEC2014-60258-C2-2-R and by the European Regional Development Fund (ERDF) as well as an Ericsson $5 \mathrm{G}$ and Tactile Internet industry grant to King's College London.

\section{REFERENCES}

1. 3GPP, "Physical Layer Procedures," TS 36.213, 3rd Generation Partnership Project (3GPP), Sept. 2009.

2. A. Ghosh and R. Ratasuk, Essentials of LTE and LTEA. Cambridge University Press, 2011.

3. 3GPP, "Non-Contiguous UL Resource Allocation: Throughput Performance," Tech. Rep. R1-091910, 3GPP TSG-RAN, Jan. 2010.

4. 3GPP, "Necessity of Multiple Bandwidths for Sounding Reference Signals," Tech. Rep. R1-074807, 3GPP TSG-RAN, Nov. 2007.

5. 3GPP, "SRS for Carrier Aggregation in LTEAdvanced," Tech. Rep. R1-100458, 3GPP TSGRAN, Jan. 2010.

6. M. Ni, X. Xu, and R. Mathar, "A Channel Feedback Model with Robust SINR Prediction for LTE Systems," in 7th European Conference on Antennas and Propagation (EuCAP), pp. 1866-1870, IEEE, 2013.

7. H. Dai, Y. Wang, K. Zhang, and C. Shi, "Link Adaptation Algorithms for Channel Estimation Error Mitigation in LTE Systems," in Global Communications Conference (GLOBECOM), pp. 1835-1840, 
Dec 2012.

8. H. Dai, Y. Wang, C. Shi, and W. Zhang, "The Evaluation of CQI Delay Compensation Schemes Based on Jakes' Model and ITU Scenarios," in 2012 IEEE Vehicular Technology Conference (VTC Fall), pp. 1-5, Sept 2012.

9. J. Heo, Y. Wang, and K. Chang, "A Novel TwoStep Channel-Prediction Technique for Supporting Adaptive Transmission in OFDM/FDD System," IEEE Transactions on Vehicular Technology, vol. 57, pp. 188-193, Jan 2008.

10. D. Martin-Sacristan, J. Cabrejas, D. Calabuig, and J. Monserrat, "MAC Layer Performance of Different Channel Estimation Techniques in UTRAN LTE Downlink," in IEEE 69th Vehicular Technology Conference, pp. 1-5, April 2009.

11. S. Falahati, A. Svensson, T. Ekman, and M. Sternad, "Adaptive Modulation Systems for Predicted Wireless Channels," IEEE Transactions on Communications, vol. 52, pp. 307-316, Feb 2004.

12. H. Sahlin, "Channel Prediction for Link Adaptation in LTE Uplink," in Vehicular Technology Conference (VTC Fall), pp. 1-5, Sept 2012.

13. J.-Y. Wu and W.-M. Lee, "Optimal Linear Channel Prediction for LTE-A Uplink Under Channel Estimation Errors," IEEE Transactions on Vehicular Technology, vol. 62, pp. 4135-4142, Oct 2013.

14. X. Xu, M. Ni, and R. Mathar, "Improving QoS by Predictive Channel Quality Feedback for LTE," in 21 st International Conference on Software, Telecommunications and Computer Networks (SoftCOM), 2013, pp. 1-5, Sept 2013.

15. F. Wang, T. Zhang, C. Feng, R. Li, and YuNgok, "Limited Feedback Scheme in the Presence of Feedback Delay using Kalman Filter," in IET International Conference on Communication Technology and Application (ICCTA 2011), pp. 253257, Oct 2011.

16. R. Akl, S. Valentin, G. Wunder, and S. Stanczak, "Compensating for CQI Aging by Channel Prediction: The LTE Downlink," in 2012 IEEE Global Communications Conference (GLOBECOM), pp. 48214827, Dec 2012.

17. K. Leung, "Power Control by Interference Prediction for Broadband Wireless Packet Networks," IEEE Transactions on Wireless Communications, vol. 1, pp. 256-265, Apr 2002.

18. F. Diehm and G. Fettweis, "Cooperative Interference Prediction for Enhanced Uplink Link Adaptation under Backhaul Delays," in IEEE 23rd International Symposium on Personal Indoor and Mobile Radio Communications (PIMRC), pp. 173-177, Sept 2012.

19. N. Mai, Y. Zakharov, and A. Burr, "Iterative B-spline Channel Estimation for Space-Time Block Coded Systems in Fast Flat Fading Channels," in IEEE 61st Vehicular Technology Conference, vol. 1, pp. 476480 Vol. 1, May 2005.
20. 3GPP, "Evolved Universal Terrestrial Radio Access (E-UTRA); Physiscal Channels and Modulation," TS 36.211, 3rd Generation Partnership Project (3GPP), Sept. 2007.

21. 3GPP, "User Equipment (UE) Radio Transmission and Reception (Release 11)," TS 36.101, 3rd Generation Partnership Project (3GPP), Sept. 2012.

22. H. Späth, One Dimensional Spline Interpolation Algorithms. Ak Peters Series, Taylor \& Francis, 1995.

23. M. A. Lema, M. Garcia-Lozano, S. Ruiz, and D. G. Gonzalez, "Improved Component Carrier Selection Considering MPR Information for LTE-A Uplink Systems," in 2013 IEEE 24th International Symposium on Personal Indoor and Mobile Radio Communications (PIMRC), pp. 2191-2196, Sept 2013.

24. M. Lema, M. Garcia-Lozano, S. Ruiz, and G. Gonzalez, "Introduction of MPR Information for Enhanced Multi-Cluster Scheduling in LTE-A Uplink," in 6th Joint IFIP Wireless and Mobile Networking Conference (WMNC), pp. 1-7, April 2013.

25. M. A. Lema, M. Garcia-Lozano, J. Olmos, and S. Ruiz, "On the Performance of LTE UL Power Control in Realistic Conditions," 4th International Conference on Mobile Networks and Management, 2012. ICTS, september 2012.

26. F. Capozzi, D. Laselva, F. Frederiksen, J. Wigard, I. Kovacs, and P. Mogensen, "UTRAN LTE Downlink System Performance under Realistic Control Channel Constraints," in IEEE 70th Vehicular Technology Conference Fall (VTC 2009-Fall), 2009, IEEE, 2009. 